\title{
Robotic Exploration with Non-Isotropic Sensors
}

\author{
Baro Hyun* Justin Jackson†, Andrew Klesh \\ Anouck R. Girard\$, Pierre Kabamba, \\ Department of Aerospace Engineering \\ University of Michigan, Ann Arbor, Michigan 48109-2140
}

\begin{abstract}
In this paper, we present an experimental implementation of an information-based path planning algorithm utilizing a three-wheeled ground robot. The robot is equipped with multiple ultrasonic sensors with which it collects information about objects of interest located within the exploration area. The mission of the robot is to identify the radius of the objects. This problem is challenging as the sensors are non-isotropic, i.e., the sensors have a limited angle of view, and the sensors are range-based, i.e., the amount of information collected by the sensors depends upon the distance from the sensor to the object of interest. The implementation shown relies on interpreting the information gathered as the inverse of uncertainty. Once a specified level of uncertainty reduction is achieved, the object is considered identified. Experimental results for a couple of different objects are provided. The results show close agreement with predicted simulations.
\end{abstract}

\section{Introduction}

Exploration has been seen as dangerous, difficult and dirty work for human explorers. However the advent of machine autonomy has increased the role of unmanned vehicles in exploration. Today, autonomous vehicles are often employed to explore an area and investigate objects of interest located within that area. The Air Force, Navy and NASA all utilize autonomous vehicles to collect information, ${ }^{9,29,35}$ and their specific roles vary application by application. For instance, in the Air Force's intelligence, surveillance and reconnaissance (ISR) missions, one goal is to gather enough information to identify whether the object of interest is a friendly object or a threatening opponent. Other examples include the Navy's underwater mine-finding mission where an unmanned underwater vehicle is used to search for underwater mines, ${ }^{9}$ and NASA's Mars exploration mission where unmanned ground vehicles are sent to investigate environmental conditions on Mars. ${ }^{35}$ Although the benefits resulting from employing unmanned vehicles in remote areas are obvious, the utilization of the vehicles is often restricted by limited time and resources. Therefore, in order to maximize the vehicle utility, it is desired to plan the vehicle trajectory in an intelligent way. Additionally, for successful mission completion, it is crucial that the path planning strategy employed take into account the information accumulation aspect in its framework in the early design phase.

Information theory is a field of study that investigates mathematical formulations of information. Shannon's seminal paper in 1948 formed the foundation of the theory, and the definition of entropy plays a central role as a measure of information, choice and uncertainty. Information-based exploration has been discussed in a few papers in recent years. ${ }^{5,17,19}$ Previously, we have posed a problem where the mission of the unmanned vehicle was to travel through a given area and collect a specified amount of information about each object of interest while minimizing the total mission time. ${ }^{24}$ Shannon's channel capacity ${ }^{36}$ was utilized as a model for information collection. An optimal control problem was stated and the necessary conditions for optimality of the path were analytically derived. Furthermore, numerical results were illustrated on several time-optimal cooperative exploration scenarios. This method was developed for vehicles

\footnotetext{
*Graduate Student, bhyun@umich.edu

†Graduate Student, jpjack@umich.edu

¥Postdoctoral Researcher, aklesh@umich.edu

$\S$ Assistant Professor, anouck@umich.edu

IProfessor, kabamba@umich.edu
} 
equipped with range-based sensors and traveling in an uncertain area. ${ }^{26}$ To reduce the uncertainty in the sensor measurement, a Kalman filter was employed where the states included the object position, visibility, and status. Moreover, a connection between Kalman filters and Shannon's equation was analytically shown through the use of a range-based covariance.

Information-collection is limited by the capability of the sensor. While sonic sensors, such as ultrasonic sensors, are capable of measuring distance, the aperture diameter is often very small to achieve high resolution at a far distance. Non-isotropic sensors are the type of sensors that information can only be collected about an object of interest that is positioned within the sensor cone. The effect of sensor non-isotropy was examined and it was shown that the width of the sensor cone affects the informative path. ${ }^{25}$

In this paper, we present an experimental implementation of an information-based path planning algorithm utilizing a three-wheeled ground robot.The contribution of this study is three-fold: 1. investigation of non-isotropic properties of the ultrasonic sensors, 2. development of an information-based exploration algorithm that identifies $n$-objects, and 3. implementation of the algorithm on unmanned ground vehicles (UGV) and comparison of the experiment results with the numerical simulation data.

The remainder of the paper is as follows. In Section II, information-theoretic measures are presented. In Section III, the vehicle kinematics and Shannon's information collection model are introduced. Additionally, the dynamic optimization problem for the path planning is formulated and the properties of the optimal path are presented. The algorithm is explained and the object identification mission is defined in Section IV. In Section V, the experiment setup is described, including the ground robot test bed, motion tracking system and the ultrasonic sensor aperture. In Section VI, experimental results and numerical simulation results are presented. Conclusions and future work are discussed in Section VII.

\section{Information-Theoretic Measures}

Information is a measure of event occurrence deduced from some statistical property directly from the event, or from some indirect observation. In this document, we briefly review some important informationtheoretic measures that are relevant to this study and highlight some of their properties.

\section{A. Shannon Information}

Entropy is a uniquely appropriate measure for evaluating information sources modeled by probabilistic descriptions. ${ }^{16}$ It is also known as a quantifier of uncertainties. ${ }^{36}$ The entropy of a discrete set of probabilities $p_{1}, \ldots, p_{n}$ is defined as:

$$
H=-\sum_{i=1}^{n} p_{i} \log p_{i}
$$

In an analogous manner, the entropy of a continuous distribution with the probability density function (pdf) $p(x)$ is defined as:

$$
H(x)=-\int_{-\infty}^{\infty} p(x) \log p(x) d x
$$

Note that $\mathrm{H}$ is always a positive value unless $p_{i}$ or $p(x)$ is equal to zero or unity. Given two complementary probabilities $p$ and $q=1-p$, the associated entropy is maximum when $p=0.5$. The implication is that when we are certain of the outcome, the entropy vanishes and when the outcome is least informative, such as uniformly distributed pdfs, maximum entropy is realized. An interesting interpretation of entropy is that it is a measure of the volume enclosed by the covariance matrix and consequently the compactness of the probability distribution. ${ }^{16}$

In a general communication system described by Shannon, the system is comprised of information source, transmitter, receiver, destination and noise source. The channel refers to the medium that is used to transmit the signal from transmitter to receiver. ${ }^{36}$ Channel capacity is a measure of transmitted information via a channel. A logarithmic scale is chosen for the channel capacity due to its functionality, as the parameters vary linearly with such scale. The choice of a logarithmic base corresponds to the choice of a unit for measuring information, hence if the chosen base is two, the resulting units may be called binary digits, or

bits. Additionally, if the logarithmic base is two, then the channel capacity is the maximum number of binary digits that can be sent per second over the channel with arbitrary small equivocation. ${ }^{36}$ 
A Gaussian white noise is a random process that has constant power spectral density. ${ }^{21}$ For a white additive noise, the channel capacity of bandwidth $W$ is

$$
C=H(y)-H(n)=W \log (1+\mathrm{SNR})
$$

where $y$ is the received signal after the noise is added to the original signal, $n$ is the noise and SNR is the signal-to-noise ratio. The signal-to-noise ratio is the ratio of the peak power signal (the peak instantaneous power of the signal) to the noise power.

\section{B. Fisher Information}

The Fisher information describes the information contained in the probability distribution function. ${ }^{16}$ The Cramer-Rao inequality can be used to give us a lower bound on the expected errors between the estimated quantities and the true values from the known statistical properties of the measurement errors. ${ }^{8}$ Given $x$ as the parameter being estimated, the Cramer-Rao inequality for an unbiased estimate $\hat{x}$ is given by

$$
P=E\left\{(\hat{x}-x)(\hat{x}-x)^{T}\right\} \geq F^{-1}
$$

where $F$ is the Fisher information matrix, ${ }^{11,12}$ which is given by

$$
F=-E\left\{\frac{\partial^{2}}{\partial x \partial x^{T}} \ln f(\tilde{y} ; x)\right\}
$$

where $f(\tilde{y} ; x)$ is the pdf of the measurement $\tilde{y}$. Note that as the Fisher information increases, the minimum covariance decreases. If the estimator is unbiased, i.e. $E\{(\hat{x}-x)\}=0$, the equality in (4) is satisfied, and the estimator is said to be efficient. ${ }^{7}$ Note that an efficient estimator is used in the information filter formulation, where the information matrix is defined as the inverse of the error covariance.

\section{Shannon \& Fisher}

The analytical properties of the two information-theoretic measures (Shannon and Fisher) differ by locality: the Shannon entropy is a global measure of the pdf, while the partial derivative on the pdf term in the Fisher information makes the measure a local one. ${ }^{14}$

Shannon's entropy is additive for mutually isolated systems which give independent data. ${ }^{14,36}$ Mutual isolation of systems allow no interaction between the subjects of measurement. An additive function is a function that preserves the addition operation, i.e. $f(x+y)=f(x)+f(y)$. Under this assumption, the additive property of Shannon as well as Fisher information can be verified, which is shown by examples in ref. 14. In ref. 2, expected entropy reduction was employed in sensor path planning and treasure hunt problem by exploiting the additive property of Shannon's information.

Shannon information and Fisher information can be identical in some cases. In ref. 24, a SNR model for active sensors was combined with Shannon's channel capacity, Eq. (3), which gives an information collection model for active sensors. The underlying assumption is that a) all sensing processes have the same bandwidth and are viewed as communication channels when operated from a single sensor, b) the visibility parameter depends on properties of the the object of interest such as the emissivity and reflectivity. The proposed model was employed in a vehicle navigation problem.

Consequently, in ref. 26 the information collection model was further explored with the use of a Kalman filter. It is shown that treating the SNR model as the variance of the measurement noise in the information filter formulation, the propagation of the information matrix is the same as the information collection model, i.e. the information can be interpreted both as Shannon's and Fisher's. The underlying assumption is that a) the SNR is small, b) the output matrix of the sensor is the square-root of the bandwidth and c) only a single vehicle is used with non-interfering object of interests. Object of interest is a subject that can be classified either as target or non-target.

\section{Information-based Path Planning}

\section{A. Modeling}

We first provide the mathematical models used throughout this paper. 


\section{The Unmanned Ground Vehicle Kinematic Model}

The UGV kinematic model is based upon the unicycle vehicle model: ${ }^{33}$

$$
\begin{aligned}
& \frac{d X}{d t}=V \cos \psi \\
& \frac{d Y}{d t}=V \sin \psi
\end{aligned}
$$

where $X$ and $Y$ are the Cartesian coordinates of the vehicle, $V$ is the fixed speed of the vehicle, and $\psi$ is the heading of the vehicle, which is the control.

\section{The Information Collection Model}

According to ref. 36 , the maximum rate at which information can be transmitted over a noisy communication channel (i.e., the channel capacity) is:

$$
\frac{d I}{d t}=W \log _{2}(1+\mathrm{SNR})
$$

where $I$ is the amount of information collected, $W$ is the sensor channel bandwidth and SNR is the signalto-noise ratio of the signal.

According to ref. 34 , a radar sensor located at Cartesian coordinates $(X, Y)$ and observing an object at Cartesian coordinates $(A, B)$ provides a signal-to-noise ratio:

$$
\mathrm{SNR}=\frac{K^{4}}{\left((X-A)^{2}+(Y-B)^{2}\right)^{2}}
$$

where the parameter $K$ depends on the object of interest.

Combining (8) and (9), the information collection model for multiple object of interests is as follows:

$$
\begin{aligned}
\frac{d I_{j}}{d t} & =W \log _{2}\left(1+\frac{K_{j}^{4}}{\left(\left(X-A_{j}\right)^{2}+\left(Y-B_{j}\right)^{2}\right)^{2}}\right) \\
1 & \leq j \leq m,
\end{aligned}
$$

where the subscript $j$ represents the $j$ th of total $m$ objects, $I_{j}$ denotes the amount of information collected from the $j$ th object and $K_{j}$ denotes a parameter that depends on the $j$ th object, which is located at Cartesian coordinates $\left(A_{j}, B_{j}\right)$. We assume that all radar sensing processes, viewed as communication channels, have the same bandwidth. Note that this model is for an isotropic sensors, which collect information at a constant rate regardless of the relative azimuth, $\Theta$. The relative azimuth is the angular distance between the vehicle to the object of interest.

A non-isotropic sensor cannot collect information at equal rates at all relative azimuths. The information collection model for non-isotropic sensor is as follows:

$$
\begin{aligned}
\frac{d I_{j}}{d t} & =\Gamma(\Theta) W \log _{2}\left(1+\frac{K_{j}^{4}}{\left(\left(X-A_{j}\right)^{2}+\left(Y-B_{j}\right)^{2}\right)^{2}}\right) \\
1 & \leq j \leq m,
\end{aligned}
$$

where $\Gamma(\Theta)$ represents the relative isotropy function that shapes the information collection rate of the sensor depending on the azimuth angle. The isotropy function is as follows:

$$
\Gamma(\Theta)=\frac{-1}{\left(1+e^{(-(\Theta-\alpha) / \beta)}\right)}-\frac{-1}{\left(1+e^{(-(\Theta+\alpha) / \beta)}\right)}
$$

where $\alpha$ is the width of the sensor cone, $\beta$ is the relative sharpness of the sensor cone and $\Theta$ is the relative azimuth between the vehicle and the object of interest. Note that sensor cones have been used to approximate non-isotropic sensors in the literature, such as in ref. 20. A pictorial description of a non-isotropic sensor coverage with varying $\beta$ can be found in ref. 25 for interested readers. 


\section{B. Path Planning Formulation and Properties}

The dynamic optimization problem is motivated by the requirement to minimize the total mission time with respect to the time-history of the heading angle of the vehicle, subject to the vehicle kinematics and information collection models, namely Eq. (6)-(11). The boundary conditions are motivated by the fact that 1) the initial position of the vehicle is known 2) the initial information with respect to objects of interest is zero 3 ) the final position of the vehicle is not constrained 4) the final informations collected must be greater than some threshold, one bit for instance. More formally,

$$
\begin{gathered}
X(0), Y(0) \text { given, } \\
I_{j}(0)=0,1 \leq j \leq m, \\
X\left(t_{f}\right), Y\left(t_{f}\right) \text { free } \\
I_{j}\left(t_{f}\right) \geq 1,1 \leq j \leq m,
\end{gathered}
$$

where $t_{f}$ denotes the final time. The necessary conditions for optimality are derived along with the costate equations: for an isotropic sensor in ref. 24, and for a non-isotropic sensor in ref. 25 for intrigued readers.

The model of information collection defined earlier gives the maximum rate of information that can be achieved with the non-isotropic sensor and a particular object of interest. By integrating forward in time along a predefined vehicle path, we can predict the amount of information collected at a given time. If we examine all the straight paths passing near an object of interest from an initial vehicle location, we can predict every point where the information boundary conditions for a specific object are met. Note that these information boundary conditions are in the problem. We informally refer to this set of points as the prediction curve, or information satisfactory bound. From analysis of the necessary conditions as well as examination of example cases, several properties of optimal paths with a non-isotropic sensor can be stated. ${ }^{25}$ Supporting illustrations of the properties can be found in ref. 25.

Property 1: When traveling between several objects of interest, if the objects of interest are isolated, then the optimal flight paths consist of sequences of straight lines (far from the objects of interest) connected by instantaneous turns (near the objects of interest).

A set of objects of interest is considered isolated if there is never a location in the space where a vehicle can simultaneously collect information about several objects at a given rate. ${ }^{24}$

Property 2: When positioned at either endpoint of the prediction curve, the angle between the vehicle's heading and the line of sight to the object of interest will be equivalent to half of the width of the sensor cone.

Property 3: When a line from the vehicle's initial position to the second object of interest passes through the prediction curve, the vehicle will travel straight to the second object of interest and collect more than the specified amount of information from the first object of interest. When the line travels through the endpoints of the prediction curve, the vehicle will travel straight to the second object of interest and collect exactly the specified amount of information from the first object of interest.

Property 4: If Property 2 does not hold, the vehicle will travel to the point on the first object of interest's prediction curve closest to the next sequential object of interest and make an instantaneous turn to travel straight to the second object.

Real-world implementation of this path planning algorithm is more complicated. While in this paper we have assumed that information accrues according to Eq. (11), often this is not the case. Additionally, we have assumed information is received at the maximum rate possible which is not always the case in reality. Furthermore, the environmental parameter $K$ is hard to quantify.

A solution to real-world implementation would be to estimate the true rate of information collection and the true value of $K .^{26}$ This would correct the information model for environmental conditions and give a better prediction of the curve for constraint satisfaction.

In the following section, we formulate a Kalman filter that correctly quantifies the information collection with respect to the objects of interest. It should be noted that this algorithm can be implemented with any sequencing method, such as the greedy method. ${ }^{6}$ This offers the flexibility of improving an optimal Traveling Salesman Problem (TSP) type path that visits each object to one that takes advantage of the prediction curve. 


\section{Software Implementation}

\section{A. Radius Estimation}

Assuming that the physical size of an object is unknown a priori, one can consider identifying the object as discovering the object size. Determining the object size may be a formidable task dependent on the mobile vehicle capability (for example, the type of sensors), and the level of certainty desired (the more precise, the more difficult). In this particular experiment, we seek to find the radius of the object within some uncertainty via ultrasonic sensors.

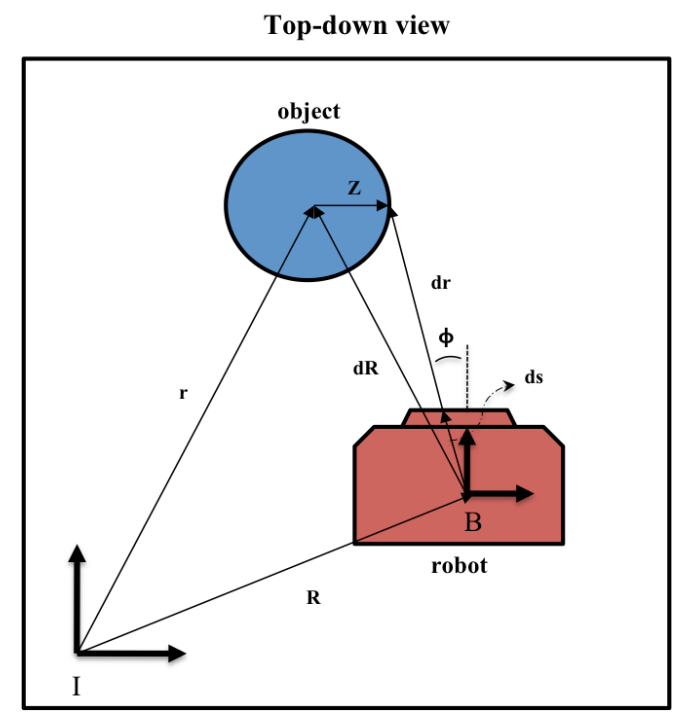

Figure 1. Radius estimation geometry

To illustrate the concept, the geometry of the vehicle, sensor, and the object is shown in Fig. 1. The inertial frame in the two-dimensional space is denoted as the $I$-frame while the vehicle body-fixed frame is denoted as the $B$-frame in the picture. The position of the vehicle and the object, $R$ and $r$, are provided via a motion tracking system. The ultrasonic sensor measures the range between the object and the vehicle, $d r$, with some level of measurement noise. Assuming that the sensor location $(d s)$ and orientation $(\psi)$ with respect to the $B$-frame are known a priori, the object radius $(Z)$ can be found by vector summation. In order to do so, reference-frame transformation as well as the misalignment of the sensor orientations must be properly addressed.

Ultrasonic sensor measures the range between the sensor and the object. Let $l$ denote the ultrasonic sensor reading. Then the range vector between the object and the vehicle, $\boldsymbol{d} \boldsymbol{r}$, measured by the sensor is obtained by

$$
\boldsymbol{d} \boldsymbol{r}=\left[\begin{array}{l}
l \cos \phi \\
l \sin \phi
\end{array}\right]
$$

where $\phi$ represents the sensor misalignment angle with respect to the centerline of the vehicle.

The two-dimensional attitude matrix $C$ that maps from the $I$-frame to the $B$-frame is defined as

$$
C=\left[\begin{array}{cc}
\cos \theta & \sin \theta \\
-\sin \theta & \cos \theta
\end{array}\right]
$$

where $\theta$ is the yaw angle between the two frames. Since we know the absolute position of the vehicle and the object as well as the sensor offset location with respect to $B$-frame, the object radius vector $\boldsymbol{Z}$ is formulated as

$$
\boldsymbol{Z}=\boldsymbol{d} \boldsymbol{R}-C^{T}(\boldsymbol{d} \boldsymbol{s}+\boldsymbol{d} \boldsymbol{r})=\left[\begin{array}{c}
X_{z} \\
Y_{z}
\end{array}\right]
$$


where $\boldsymbol{d} \boldsymbol{R}=\boldsymbol{r}-\boldsymbol{R}$ is the range vector from $B$-frame to the center of the object, $\boldsymbol{d} \boldsymbol{s}$ denotes the sensor offset location from the origin of $B$-frame, superscript $T$ denotes the transport operator and $X_{z}, Y_{z}$ represent the $\mathrm{x}, \mathrm{y}$ components of the radius vector. The scalar radius is the Euclidean norm of the radius vector, $Z=\|\boldsymbol{Z}\|=\sqrt{X_{z}^{2}+Y_{z}^{2}}$

Due to the presence of noise in the measurement, we cannot expect to have perfect knowledge of the object radius. In order to alleviate the effect of noise, a Kalman filter is employed to provide a real-time state estimate of the object radius and its associated state-estimate covariance. Note that this particular Kalman filter accounts for the range-dependent measurement noise. The detailed mathematical development of a Kalman filter for active range-based sensors can be found in ref. 23.

Here we assumed that the noises in the robot and object position are small.

As the error covariances of the estimate and information are reciprocal, the covariance provided by the filter is used as a measure of information. Note that in this case the information is interpreted as in the sense of Fisher, which we have discussed earlier in Section II. Note that the covariance is an important parameter in this study since it determines whether the vehicle should continue collecting additional information or not.

\section{B. The Algorithm}

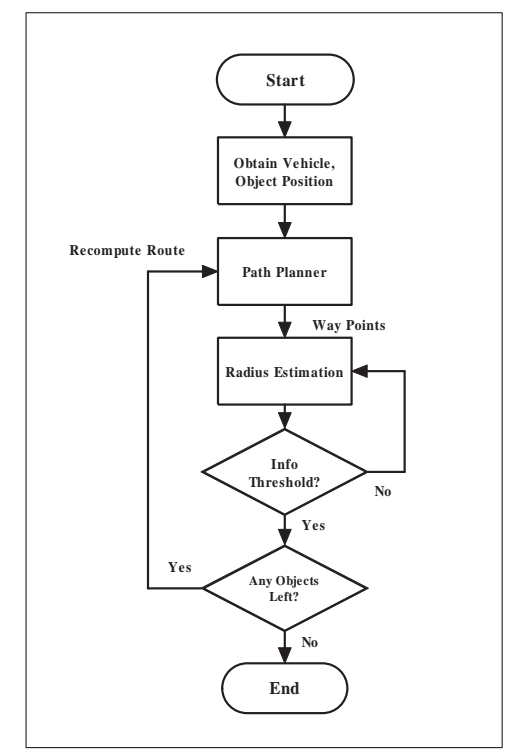

Figure 2. Flow chart of the algorithm

Fig. 2 shows the algorithm flow chart that was implemented for this experiment. First, the initial position of the vehicle and the position of $n$ objects are obtained through the motion tracking system, which is described in the next session. The position information is employed by the path planner algorithm, and the algorithm generates a trajectory toward the object of interest that the robot should follow. A greedy algorithm, based on the distance between the vehicle and the object, is employed to determine which object the vehicle should visit first.

The path planner provides a predicted location of where the information threshold will be met (satisfaction boundary) based on the actual sensor aperture of the vehicle. Prior to vehicle movement, the onboard ultrasonic sensor is activated. While the vehicle is following the generated path, the sensor measures the range between the object and the vehicle and an estimate of the object radius, as well as its error covariance, are computed in real-time. Note that if the object is not within the sensor cone for some period of time, the covariance is constant during the time, since no information is received.

A covariance threshold is set as an indication of sufficient information. Once the covariance reduces down to the threshold, the vehicle stops and checks the existence of any additional objects to visit. In case of multiple objects, the path planner recalculates the route. If the covariance threshold is not met, the vehicle 
continues to follow the path until it collects enough information.

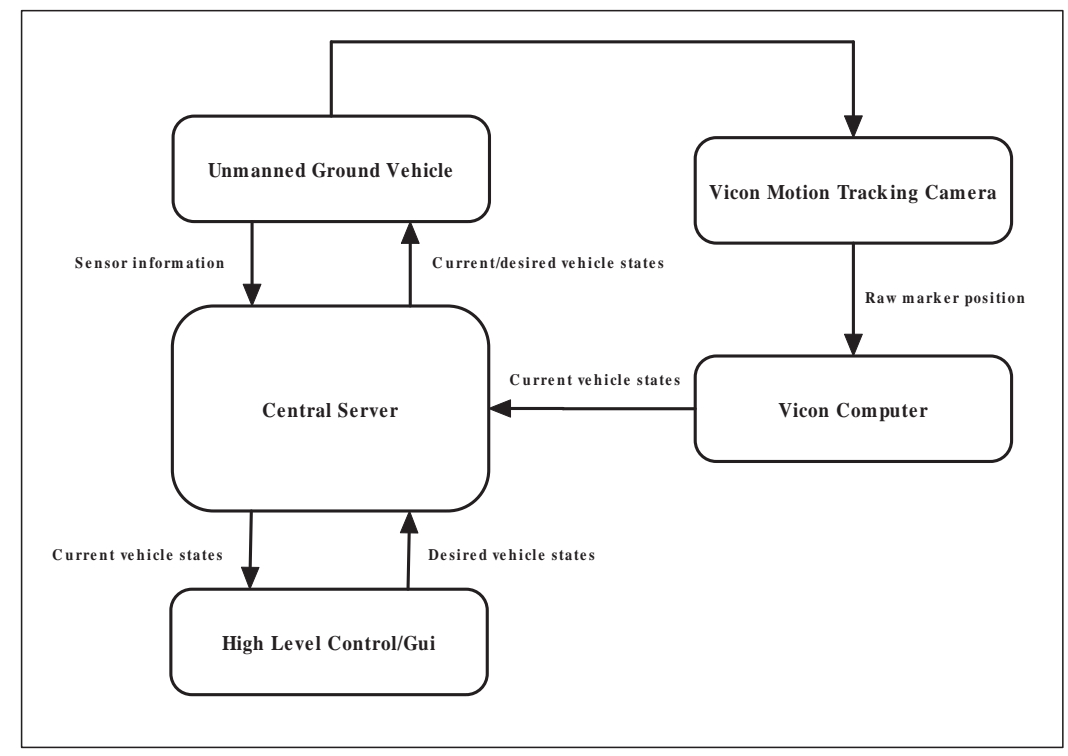

Figure 3. Data flow chart

\section{Hardware Setup}

An Amigobot ${ }^{T M}$, a mobile unmanned ground vehicle equipped with multiple onboard ultrasonic sensors, was used as the robot testbed. The Amigobot, from Mobile Robots, Inc., is a three-wheeled robot that has eight range-finding ultrasonic sensors: six forward and two rear. It has twin 500-tick motor encoders, a Renesas SH7144-driven microcontroller, dual serial ports, and 1Mb onboard RAM. The maximum payload is one kilogram and the maximum speed is one meter per second. The robot is powered by a $12 \mathrm{~V}$ sealed lead-acid battery and a fully loaded AmigoBot base can run up to 2 hours at full charge. The robot is capable of receiving waypoint commands as well as sensor activation commands in real-time through wireless communication and the real-time sensor reading is sent in packets to the mother PC. The computing processor located on top of the Amigobot is a Pentium $1 \mathrm{GHz}$ with Ubuntu.

A motion tracking system, from Vicon, Inc., was employed for tracking the vehicle as well as the object. Vicon MX $-3+$ cameras record $659 \times 493$ full frame grayscale pixels at the speed of 120-130 frames per second. The tracking precision of the system is in the sub-millimeter scale. At total of seven cameras are placed at the corner and edges in the Aerospace Robotics and Control laboratory (ARC lab) at the University of Michigan. The total tracking coverage is a circular area with approximately a meter radius.

Fig. 3 shows the overview of the data flow between the server, the motion tracking system, control algorithm source and the mobile vehicle. The tracking information is transferred to the central server in real-time.

\section{A. Non-isotropic Sensor Aperture}

We refer to the volume that the sensor can cover as the sensor cone. An object located within this cone transmits information. There are six ultrasonic sensors in the front of the vehicle. They are distributed symmetrically from the vehicle center line with offset angles given as

$$
\psi=\left[\begin{array}{llllll}
90 & 52.9 & 20.3 & -20.3 & -52.9 & -90
\end{array}\right](\mathrm{deg})
$$

The ultrasonic transmitters emit sound waves via a circular emitter. The circular emitter has a diameter of $52 \mathrm{~mm}$ and the waves form a trapezoid-like shape with a deviation angle of $4.26 \mathrm{deg}$. Fig. 4 shows a picture of the ultrasonic sensor emitters and the sensor cone. Furthermore, the sensor collects information at 


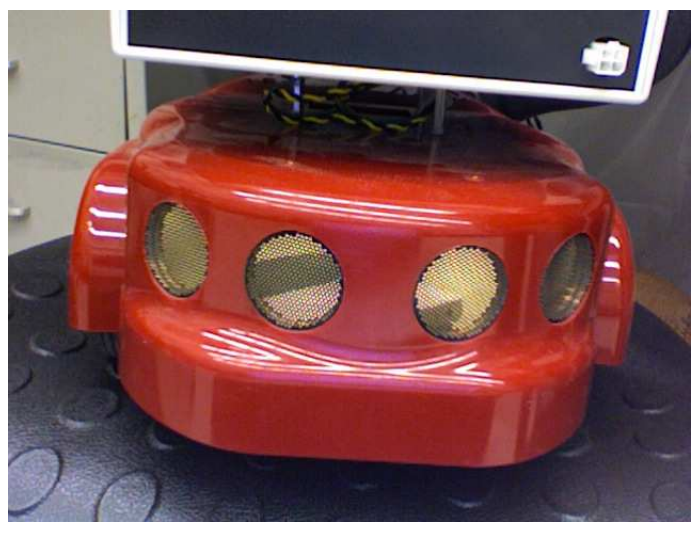

(a) Sensor emitter outlet

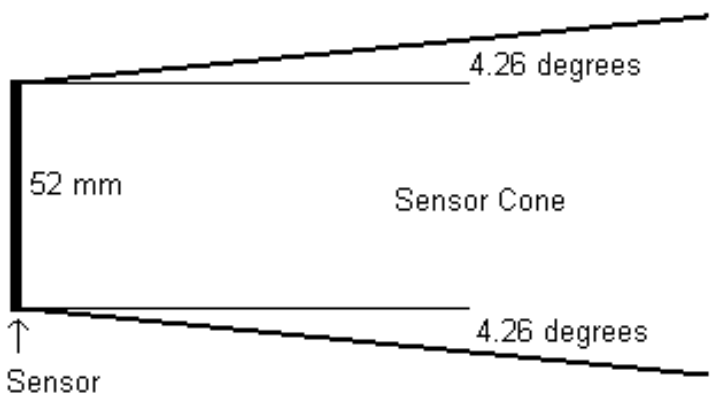

(b) Sensor cone

Figure 4. Ultrasonic sensor aperture

Top-down view

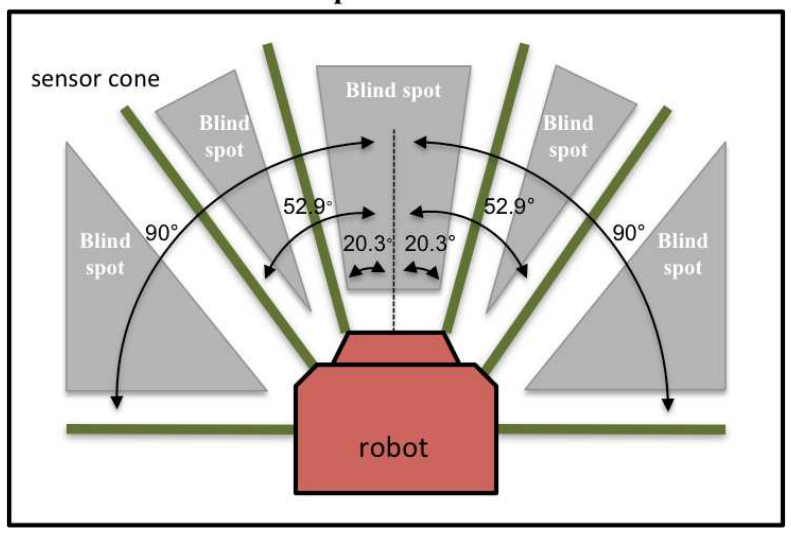

(a) Unmodified sensor configuration
Top-down view

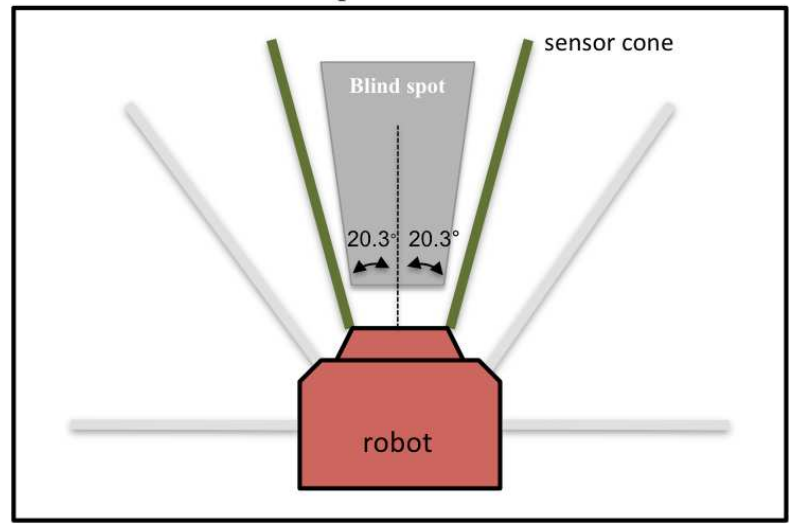

(b) Modified sensor configuration

Figure 5. Sensor orientations and blind spots

maximum rate when the object is placed in the center of the sensor cone (within $52 \mathrm{~mm}$ diameter cylinder) while as the object deviates from the center the collection rate reduces, due to the deflection of the waves. Therefore, we refer to the sensor as non-isotropic. Each sensor has a cone length of about 6.8 meters. The range readings from the sensors are sampled with the rate of $50 \mathrm{~Hz}$.

Due to the alignment of the sensor orientations, there are multiple blind spots from which the sensor cannot measure the object. The presence of multiple blind spots is undesired since the non-isotropic sensor model, Eq. (12), does not take them into account. Thus, we use the front two sensors that are closest to the vehicle center line and reduce the number of blind spots. The concept is illustrated in Fig. 5.

\section{B. Object}

The objects of interest are stationary and have a cylinder-like form with constant radius. Two different types of structures were considered: a full-body cylinder and an empty-body cylinder. A full-body cylinder structure has a solid surface around the body and is made of metal. An empty-body cylinder structure has a pole that supports the top and bottom part of the structure while the body part is empty except for the pole inside. (Fig. 6 (a)) Instead of metal, the structure is covered with a cotton material that makes a loose-surface, as oppose to the previous solid-surface. The loose-surface cylinder gives a slightly degraded SNR measurement with respect to the solid-surface cylinder.

According to Eq. (9), the signal to noise ratio of a signal is proportional to the parameter $K$. This parameter can vary depending on the environmental conditions such as object surface. While it is hard to 
identify the actual $K$, by correctly modeling the vehicle and object positions and the noise properties of the signal, a rough estimate can be computed via numerical fitting. Fig. 6 (b) shows one example.

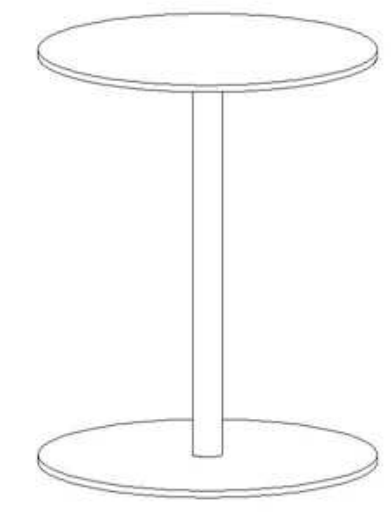

(a) Empty-body cylinder structure

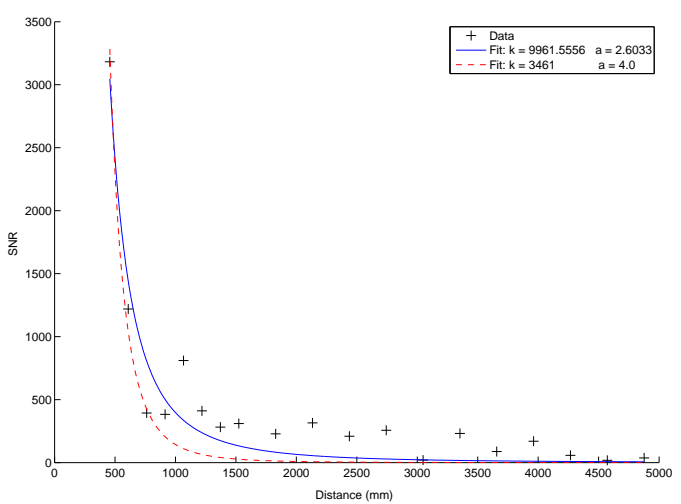

(b) Estimate of parameter $K$ by numerical fitting

Figure 6. Object structure and parameter $K$

\section{Experiment Results}

The initial robot position and orientation were chosen randomly in each trial. The path optimization was run on an Apple Macbook labtop and the computed path was sent back to the robot via wireless communication. In the experiment, two objects were used and the their position was randomly chosen. For demonstration purposes, the empty-body object was placed closer to the robot's initial position so that the robot visits the empty-body object before the full-body object.

Fig. 7 shows the vehicle trajectory in Cartesian coordinates and the time history of the error covariance for one of the trials. In Fig. 7 (a), the information-satisfaction bound (denoted in dots) was computed using the actual ultrasonic sensor aperture. This satisfaction bound is computed numerically, and it may not give the sufficient amount of information. In Fig. 7 (b), the red line denotes the threshold that the covariance should reach in order to proceed to the next object. Once the covariance has reached the threshold, the initial covariance value is reset. The figure shows that the covariance for each object was successfully reached the threshold. Another result with different set up is shown in Fig. 8.

The repeatability of the experiment is described in the conclusion.

\section{Conclusion}

An information-based path planning algorithm that accounts for non-isotropy of the sensor was validated via a three-wheeled ground vehicle testbed with onboard ultrasonic sensors. The algorithm enables the vehicle to travel through a given exploration area and collect a specified amount of information about the state of each object of interest while minimizing the total mission time. The experiment results showed close agreement with the numerical simulation results.

Disagreement between the simulated path and the experimental results were exhibited in some cases. The authors believe that the disagreement is due to the following reasons. First, a wrong $K$ value can cause a significant change in the information satisfactory bound, which can affect the length of the straight portion of the path. Second, the sensor blind spots are not precisely captured in the non-isotropic sensor model. Therefore, the generated path cannot predict when the object of interest will fall into the blind spot. To the best of our knowledge, as a good estimate of $K$ value is at hand and the effect of the sensor blind spots is take into account, the experiment is repeatable with comparable performance.

In future work, we are planning to change the sensor configuration so that the blind spot within the sensor aperture is removed. This can be done by relocating the two sensors on a flat surface, for example a small board, and attaching it in the front part of the robot. By doing so, improvement in measurement 


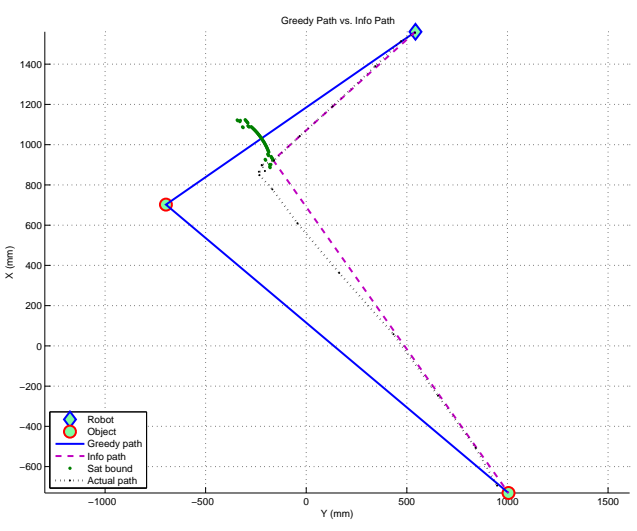

(a) Path generated compared to the actual path

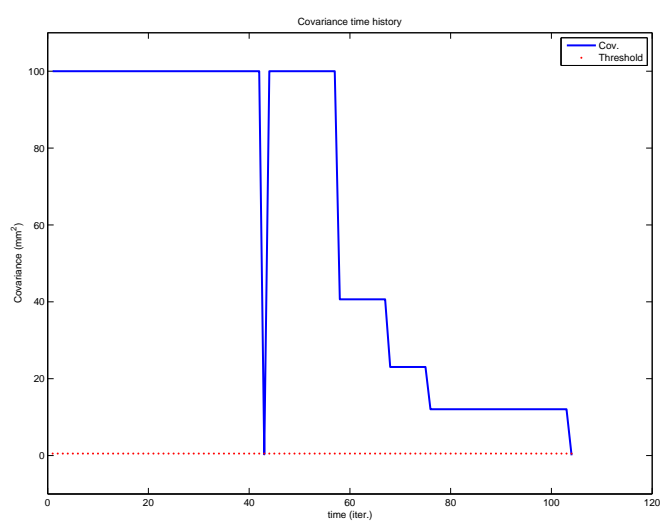

(b) Covariance time history. The solid blue line indicates the actual covariance (starts from $100 \mathrm{~mm}^{2}$ ) while the dotted red line indicates the threshold $\left(0.5 m m^{2}\right)$.

Figure 7. Trial 1

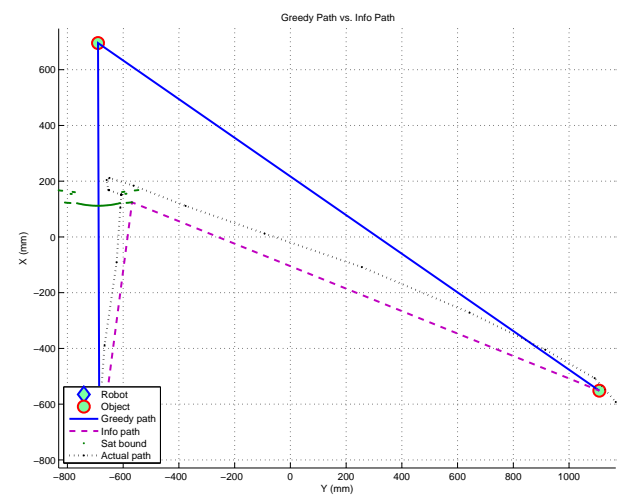

(a) Path generated compared to the actual path

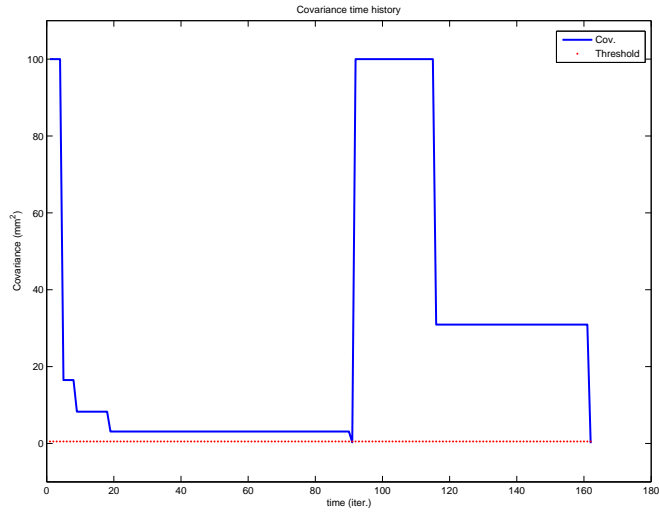

(b) Covariance time history. The solid blue line indicates the actual covariance (starts from $100 \mathrm{~mm}^{2}$ ) while the dotted red line indicates the threshold $\left(0.5 \mathrm{~mm}^{2}\right)$.

Figure 8. Trial 2

consistency is expected. A cooperative exploration scenario with multiple vehicle and objects is our ultimate goal.

\section{References}

\footnotetext{
${ }^{1}$ Baumgartner, K. and Ferrari, S., "Optimal placement of a moving sensor network for track coverage," Proceedings of the American Control Conference, New York City, NY, Jul. 2007.

${ }^{2}$ Cai, C., "Information-driven sensor path planning and the treasure hunt problem," Doctoral dissertation, Department of mechanical engineering and materials science, Duke university, 2008

${ }^{3}$ Cai, C., Ferrari, S., and Qian, M., "Bayesian network modeling of acoustic sensor measurements," IEEE Sensors Conference, pp.345-348, 2007.

${ }^{4}$ Cai, C., and Ferrari, S., "Comparison of information-theoretic objective functions for decision support in sensor systems," Proceedings of the American Control Conference, NYC, NY, Jul. 11-13, 2007.

${ }^{5}$ Chung, T., Gupta, V., Burdick, J., and Murray, R., "On a decentralized active sensing strategy using mobile sensor platforms in a network," IEEE Conf. on Decision and Control, pp. 1914-1919, 2004.
} 
${ }^{6}$ Cormen, T.H., Leiserson, C.E., Rivest, R.L., and Stein, C., "Introduction to Algorithms," 3rd edn, The MIT Press, 2009.

${ }^{7}$ Cramér, H., "Mathematical methods of statistics," Princeton University Press, Princeton, NJ, 1946.

${ }^{8}$ Crassidis, J.L., and Junkins, J.L., "Optimal Estimation of Dynamic Systems," Chapman Hall/CRC Press, Boca Raton, FL, 2004

${ }^{9}$ Fernandez, J., Christoff, J., Cook, D., Station, C., Center, N., and Spring, S., "Synthetic aperture sonar on AUV," OCEANS 2003. Proceedings, Vol. 3, 2003.

${ }^{10}$ Fierro, R., Ferrari, S., and Cai, C., "An information-driven framework for motion planning in robotic sensor networks: complexity and experiments," Proceedings of the 47th IEEE Conference on Decision and Control, Cancun, Mexico, Dec. 2008.

${ }^{11}$ Fisher, R.A., "Contribution to mathematical statistics (collection of papers published 1920-1943)," Wiley, New York, NY, 1950.

${ }^{12}$ Fisher, R.A., "Statistical methods and scientific inference", Hafner Press, New York, NY, 3rd ed., 1973.

${ }^{13}$ Frieden, B.R., "Probability, statistical optics and data testing," 2nd edn, Springer-Verlag, Berlin, 1991.

${ }^{14}$ Frieden, B.R., "Physics from Fisher information," Cambridge University Press, Cambridge, UK, 1998.

${ }^{15}$ Grime, S. and Durrant-Whyte, H.F., "Data fusion in decentralized sensor networks," Control Eng. Practice, Vol.2, No.5, pp. 849-863. 1994

${ }^{16}$ Grocholsky, B., "Information-theoretic control of multiple sensor platforms," Doctoral dissertation, Department of aerospace, mechatronics and mechanical engineering, The university of Sydney, Mar. 2002

${ }^{17}$ Grocholsky, B., Makarenko, A., and Durrant-Whyte, H., "Information-theoretic coordinated control of multiple sensor platforms," Robotics and Automation, 2003 Proceedings. ICRA'03. IEEE International Conference on, Vol. 1, 2003

${ }^{18}$ Hero, A.O., Ma, B., Michel, O., Gorman, J.D., "Alpha divergence for classification, indexing and retrieval," Technical Report 328, Communications and Signal Processing Laboratory (CSPL), Department of EECS, University of Michigan, Ann Arbor, May, 2001. 2008.

${ }^{19}$ Hussein, I., "Kalman filtering with optimal sensor motion planning," Proceedings of the American Control Conferece,

${ }^{20}$ Jaradat, K. and Langari, R. "Line map construction using a mobile robot with a sonar sensor," Advanced Intelligent Mechatronics. Proceedings, 2005 IEEE/ASME International Conference on, pages 1251-1256, 2005.

${ }^{21}$ Kabamba, P., "Guidance, navigation and avionics," Lecture notes, To be published.

${ }^{22}$ Kalman, R.E. and Bucy, R.S., "New results in linear filtering and prediction theory," Journal of Basic Engineering, pp. 95-108., Mar. 1961

${ }^{23}$ Klesh, A., Girard, A., Kabamba, P., "Real-time path planning for time-optimal exploration," In Proceedings of the 2008 AIAA GNC conference, Aug. 2008.

${ }^{24}$ Klesh, A., Girard, A., Kabamba, P., "Path planning for cooperative time-optimal information collection," In 2008 American Control Conference, Jun. 2008.

${ }^{25}$ Klesh, A., Huntsberger, T., Woodward, G., Kabamba, P., and Girard, A., "Tactical area search with non-isotropic sensors," Jet Propulsion Laboratory, 2008.

${ }^{26}$ Klesh, A., Kabamba, P., Girard, A., "Optimal path planning for uncertain exploration," In 2009 American Controls Conference, Jun. 2009.

${ }^{27}$ Kreucher, C., Kastella, K., and Hero, A.O., "Sensor management using an active sensing approach," Signal Processing, 85:608-624, 2005.

${ }^{28}$ Kullback, S., "Information theory and statistics," Wiley, New York, 1959.

${ }^{29}$ Office of the Secretary of Defense, "Unmanned Aircraft Systems Roadmap, 2005-2030,"

${ }^{30}$ Principe, J., Xu, D., and Fisher, J., "Information-theoretic learning," In S. Haykin, editor, Unsupervised Adaptive Filtering, Wiley, pp. 265-319, 1999.

${ }^{31}$ van der Meulen, E., "A survey of multi-way channels in information theory:1961-1976," IEEE Transactions on Information Theory, 23(1):1-37, 1977.

${ }^{32}$ Rényi, A., "On measures of entropy and information," In the Fourth Berkeley Symposium on Mathematical Statistics and Probability, vol. 1, pp. 547-561. 1961.

${ }^{33}$ Savla, K., Bullo, F., and Frazzoli, E., "On traveling salesperson problem for Dubins' vehicle," In Proceedings of the 44th IEEE Conference on Decision and Control and the European Control Conference 2005, IEEE. 2005.

${ }^{34}$ Scheler, D., "Introduction to Electronic Warfare," 1986.

${ }^{35}$ Schenker, P., Huntsberger, T., Pirjanian, P., Baumgartner, E., and Tunstel, E., "Planetary Rover Developments Supporting Mars Exploration, Sample Return and Future Human-Robotics Colonization", Autonomous Robots, Vol. 14, No. 2, 2003, pp. $103-126$

${ }^{36}$ Shannon, C., "A Mechanical Theory of Communication," The Bell System Technical Journal, 27:379-423, 623-656, 1948. 